\title{
Desempeño pedagógico docente y aprendizaje de los estudiantes universitarios en la carrera de Educación
}

\author{
Llesica Soria Ramirez $^{\left.1{ }^{(}\right)}$, Wilmer Ortega Chávez ${ }^{(0)}$, Arnulfo Ortega Mallqui ${ }^{(\mathbb{1}}$ \\ Universidad Nacional de Ucayali, Universidad Nacional Intercultural de la Amazonia, Universidad Nacional Hermilio Valdizán, Perú
}

Autor de correspondencia: llesicarsoria@gmail.com Recibido: 02 de enero de 2020 Revisado: 30 marzo de 2020 Aprobado: 01 de julio de 2020 Publicado: 16 de agosto de 2020

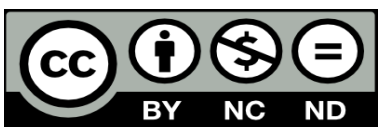

\section{Resumen}

El estudio tuvo como objetivo determinar la relación entre el desempeño docente y el aprendizaje de estudiantes de las carreras profesionales de Educación Inicial y Educación Primaria, durante el segundo semestre de 2016, los cuales ingresaron en 2014 a la Facultad de Educación y Ciencias Sociales de la Universidad Nacional de Ucayali-Pucallpa. El diseño de la investigación fue no experimental, transversal y descriptivocorrelacional. La muestra estuvo conformada por 94 estudiantes de los programas mencionados. Se aplicaron dos instrumentos. Con uno se midió el desempeño docente, dividido en cuatro dimensiones -enseñanza-tutoría, proceso de enseñanzaaprendizaje, investigación y extensión universitaria-proyección social- segmentadas en 27 ítems con escala tipo Likert. Con el segundo instrumento se midió el aprendizaje, dividido en tres dimensiones -enfoque superficial, enfoque profundo y enfoque de alto rendimiento- segmentadas en 33 ítems con escala tipo Likert. La conclusión fue que existe un alto grado de correlación entre el desempeño docente y el aprendizaje de los estudiantes, con lo que se infiere que el desempeño docente influye de manera positiva en el aprendizaje.

Palabras claves: educación, proceso de aprendizaje, desempeño docente, estudiante universitario, enseñanza 


\title{
Teaching pedagogical performance and learning of university students in the Education career
}

\begin{abstract}
The main objective of the study was to determine the connection between teaching performance and the learning of students of the majors in Early Education and Primary Education, during the second semester of 2016, who joined to the Faculty of Education and Social Sciences of the National University of Ucayali-Pucallpa in 2014. The research design was non-experimental, transversal, and correlational-descriptive. The sample consisted of 94 students of the two programs. Two instruments were applied. The first one helped to measure the teacher performance, which was divided into four dimensions-teachingtutoring, teaching-learning process, research, and university extension-social projectionsegmented in 27 items with a Likert type scale. The second instrument was used for measuring the learning, which was divided into three dimensions-superficial perspective, deep perspective, and high-performance perspective- segmented in 33 items with a Likert type scale. The conclusion was that there is a high degree of correlation between teacher performance and student learning, thus inferring that teacher performance positively influences learning.
\end{abstract}

Keywords: education, learning process, teaching performance, university student, teaching

\section{Atuação pedagógica docente e aprendizagem de universitários na carreira de Educaçãoa}

\section{Resumo}

O objetivo deste estudo foi determinar a relação entre o desempenho docente e a aprendizagem dos alunos dos programas de Educação Infantil e Educação Básica, durante o segundo semestre de 2016, os quais entraram em 2014 à Faculdade de Educação e Ciências Sociais da Universidade Nacional de Ucayali-Pucallpa. O desenho da pesquisa é não experimental, transversal e descritiva-correlacional. A amostra foi constituída por 94 alunos dos dois programas. Foram aplicados dois instrumentos. Com o primeiro se avaliou o desempenho do professor, dividido em quatro dimensões - ensino-mentoria, processo de ensino-aprendizagem, pesquisa e extensão universitária - projeção social segmentadas em 27 itens com escala do tipo Likert. Com o segundo instrumento se mediu a aprendizagem, a qual foi dividida em três dimensões - foco superficial, foco profundo e foco de alto desempenho - segmentadas em 33 itens com escala do tipo Likert. A conclusão foi que há muita correlação entre o desempenho do professor e a aprendizagem do aluno, inferindo assim que o desempenho do professor influencia positivamente a aprendizagem.

Palavras-chave: educação, processo de aprendizagem, desempenho docente, estudante universitário, ensino 
En la universidad no solo se le exige más al estudiante, también hay exigencias que debe cumplir el docente, ya que su nivel de educación debe ser profesional, especializado y acorde con la carrera profesional universitaria que cursa el alumno. Esas exigencias son evaluadas a través de su desempeño como docente - conducta que asume durante el proceso de enseñanza y que está enmarcado en la conducta real del mismo; lo que en realidad se ejecuta-. El desempeño puede verse afectado por el cansancio y por factores emocionales o psicológicos, entre otros. A través de su desempeño, el docente asume un compromiso para garantizar la adecuada preparación de futuros profesionales.

Según Rueda (2009), el desempeño docente es "la capacidad de enfrentar demandas complejas, apoyándose en los recursos psicosociales, incluyendo las destrezas y actitudes en un contexto en específico y cómo este maneja las herramientas disponibles, tanto físicas como socioculturales" (p. 3).

Antes de ahondar en este tema, es preciso conocer la realidad de las carreras profesionales de Educación Inicial y Educación Primaria en el Perú. Una encuesta del Instituto Nacional de Estadística e Informática (2014), realizada a 10564 egresados universitarios de entre 21 a 30 años, arrojó que el 3,2\% de egresados de Educación Inicial y de Educación Primaria tienen trabajo, con ingresos económicos de 1400 nuevos soles mensuales -US\$ 424-. Respecto a su percepción sobre el desempeño de los docentes de la universidad, $1933-18,3 \%$ indicaron que los docentes eran de calidad, tanto en las universidades públicas como en las privadas. Esta encuesta es un antecedente sobre el desempeño docente universitario, el mismo que actualmente es evaluado con fines de calidad por el Sistema Nacional de Evaluación, Acreditación y Certificación de la Calidad Educativa.

Tradicionalmente, la investigación sobre la función de producción de la educación ha sido enfocada de forma empírica. En ella se busca determinar cómo los profesores y sus características de fondo contribuyen al rendimiento de los estudiantes, con base en pruebas estandarizadas (Bazar \& Kraft, 2017). Sin embargo, es evidente el aspecto multidimensional del aprendizaje de los estudiantes, con factores e indicadores que van más allá del mero conocimiento académico de los docentes (Duckworth et al., 2012). Las dimensiones del aprendizaje y los sistemas de creencias académicas son factores significativos que contribuyen al logro académico. La calidad de la educación superior solo se puede mejorar si se estudian sus efectos en los estudiantes, en un sistema basado en el desempeño de sus profesores (Usun, 2004). Por lo tanto, para determinar los factores esenciales que afectan el aprendizaje y el rendimiento académico de los estudiantes, es fundamental su opinión y su percepción.

Es bien conocido que el proceso de aprendizaje de los estudiantes universitarios depende de diversos factores, por lo que, para medir su nivel de aprendizaje, se debe hacer un análisis de los diferentes enfoques de aprendizaje.

El origen de los diferentes enfoques de aprendizaje puede rastrearse hasta los primeros años de la década de los setenta del siglo pasado, en la Universidad de Gotemburgo Suecia-, donde se gestaron las teorías básicas de las diferentes formas como los estudiantes afrontan tareas de aprendizaje. De allí surgen los conceptos de los enfoques superficial y profundo, categorías que han permanecido hasta la actualidad (Soler-Contreras et al., 2017). El enfoque superficial puede definirse como la estrategia en la que el estudiante 
memoriza la información como hechos aislados, sin establecer conexión alguna con experiencias previas o con su contexto general (Fasce, 2007). En este enfoque, el objetivo central es retener datos para aprobar una evaluación, por lo que se adquiere un bajo nivel cognitivo y un rápido olvido de lo estudiado. Por otro lado, el enfoque profundo se refiere a la incorporación del análisis crítico de las ideas, que son integradas al conocimiento previo sobre el tema, lo que favorece su comprensión y su retención (Fasce, 2007). Este aprendizaje promueve la comprensión de lo estudiado y su aplicación en los aspectos de la vida donde sea necesario. El enfoque de aprendizaje basado en el alto rendimiento agrupa algunos aspectos de los enfoques superficial y profundo. En él se define a una minoría de estudiantes que se caracteriza por aspirar a las calificaciones máximas como objetivo principal en su aprendizaje (Muñoz \& Gómez, 2005). Este último enfoque de aprendizaje cada vez se usa menos, por la tendencia a utilizar el enfoque profundo como medio para alcanzar el mayor rendimiento y la mayor comprensión. Sin embargo, aunque en porcentaje bajo, todavía se encuentran estudiantes que se inclinan por alcanzar altos rendimientos.

Se puede decir, entonces, que un enfoque de aprendizaje es el camino que sigue un estudiante al enfrentarse a una demanda académica en el ámbito educativo y que él mismo está mediado por la motivación a aprender y por las estrategias en sí (Soler et al. 2018). En el caso de la educación formal, el aula de clase es el espacio donde suceden estos eventos. De allí que hayan surgido numerosas investigaciones enfocadas en develar las relaciones de enseñanza y aprendizaje que se dan entre estudiantes y docentes, como actores principales del proceso educativo (Acosta, 2017; Díaz \& Pérez, 2013; Gálvez \& Milla, 2018; Gómez et al., 2011; Monroy, 2012; Palomino, 2012; Rahmatullah, 2016).

La presente investigación tiene como objetivo mostrar la relación entre el desempeño docente y el aprendizaje en los estudiantes de las carreras profesionales de Educación Inicial y de Educación Primaria de la Facultad de Educación y Ciencias Sociales de la Universidad Nacional de Ucayali. Para ello, se tiene como premisa lo mencionado por Freire y Faundez (2013) acerca de que el asunto del maestro no es de saber qué enseñar, sino cómo enseñar, qué estrategias didácticas emplear para que nuestros estudiantes asimilen la información y puedan actuar ante un mundo cambiante y vertiginoso, donde la información tiene cada vez más un lugar primordial en la sociedad. El éxito del docente es saber cómo emplear los medios tecnológicos adecuados para el proceso de enseñanza y aprendizaje en los estudiantes.

Esta investigación parte de una visión instrumental de la educación, concebida desde un saber socioeconómico a través de la creación de un sistema educativo que genera estudiantes enfocados en un alto rendimiento para generar mejores beneficios económicos después de egresados.

\section{Metodología}

Según el propósito de la investigación, el diseño que más se ajustó al estudio fue el no experimental, de tipo descriptivo-correlacional. La población de estudio estuvo conformada por todos los estudiantes - un total de 94, entre hombres y mujeres- matriculados en el semestre 2016-II de las carreras profesionales de Educación Inicial y de Educación Primaria de la Facultad de Educación y Ciencias Sociales de la Universidad Nacional de Ucayali de la 
ciudad de Pucallpa en Perú. Se trabajó con un muestreo no probabilístico, ya que se tomó el $100 \%$ de la población, debido a que es finita, de modo que se pudieran obtener resultados más confiables.

El instrumento utilizado permitió el registro de cómo se viene trabajando el desempeño docente y, gracias a ello, cómo se genera el aprendizaje en los estudiantes. La variable desempeño docente contó con 27 ítems y cuatro dimensiones: enseñanza-tutoría, proceso de enseñanza-aprendizaje, investigación y extensión universitaria-proyección social. La variable aprendizaje tuvo 33 ítems y tres dimensiones: enfoque superficial, enfoque profundo y enfoque de alto rendimiento. Para obtener la información, cada dimensión fue segmentada en indicadores, los cuales fueron redactados con un lenguaje comprensible para que el encuestado contribuyera a la investigación. La escala de medición de cada ítem fue de un punto como mínimo y de tres puntos como máximo, en una escala de tipo Likert que registra datos cualitativos de: nunca, a veces y siempre.

La validación del contenido del instrumento se hizo mediante el juicio de expertos, que analizaron su consistencia. Para la confiabilidad, se aplicó una prueba piloto a veinte estudiantes de las carreras de Economía y Negocios Internacionales de la misma universidad. Se empleó el Alpha de Cronbach, el cual dio como resultado un valor de 0.957, lo cual representó una alta confiabilidad. Para el análisis de la información, se utilizó la prueba de correlación de Pearson y el programa estadístico SPSS, en su versión 23. El coeficiente de correlación de Pearson sirvió para analizar el nivel de relación que puede existir entre los indicadores de estudio, los cuales definen las variables desempeño docente y aprendizaje.

\section{Resultados}

En la tabla 1, se muestran los resultados descriptivos obtenidos para las variables globales, desempeño docente y aprendizaje de los estudiantes de la muestra del estudio.

\section{Tabla 1}

Resultados descriptivos de las variables desempeño docente $y$ aprendizaje

\begin{tabular}{llllll}
\hline \multicolumn{2}{l}{ Desempeño docente } & \multicolumn{4}{c}{ Aprendizaje } \\
\multirow{2}{*}{ Malo } & Recuento esperado & 0.5 & 2.6 & 1.9 & 5.0 \\
& \% del total & $4.3 \%$ & $1.1 \%$ & $0.0 \%$ & $5.3 \%$ \\
\multirow{2}{*}{ Regular } & Recuento esperado & 3.2 & 15.3 & 11.5 & 30.0 \\
& \% del total & $5.3 \%$ & $24.5 \%$ & $2.1 \%$ & $31.9 \%$ \\
\multirow{2}{*}{ Bueno } & Recuento esperado & 6.3 & 30.1 & 22.6 & 59.0 \\
& \% del total & $1.1 \%$ & $25.5 \%$ & $36.2 \%$ & $62.8 \%$ \\
\multirow{2}{*}{ Total } & Recuento esperado & 10.0 & 48.0 & 36.0 & 94.0 \\
& \% del total & $10.6 \%$ & $51.1 \%$ & $38.3 \%$ & $100.0 \%$ \\
\hline
\end{tabular}

En la tabla 1, se observa que la mayoría de los estudiantes - 36.2\% — calificó el desempeño docente como bueno, y a su vez marcó la alternativa siempre respecto al aprendizaje; mientras que el 1.1\% indicó que el desempeño docente es malo, y a su vez marcó la alternativa regular respecto al aprendizaje adquirido. Lo anterior se puede interpretar como una percepción positiva, relacionada con la tendencia de los docentes a propiciar y potenciar el aprendizaje 
entre los estudiantes, lo que se puede traducir en motivación y adquisición de conocimiento significativo.

En la tabla 2, se muestran los resultados entre la variable desempeño docente y la dimensión enfoque superficial. La prueba de correlación de Pearson muestra como resultado $r=-0.335$, lo que indica que entre el desempeño docente y la dimensión enfoque superficial del aprendizaje de los estudiantes existe un grado de correlación moderada (Hopkins, 2014) e inversamente proporcional, lo que indica que el desempeño docente no influye en gran forma sobre la tendencia de los estudiantes a utilizar el enfoque superficial dentro de su aprendizaje.

Tabla 2

Resultados del desempeño docente y la dimensión enfoque superficial

\begin{tabular}{llllll}
\hline \multicolumn{5}{c}{ Enfoque Superficial } \\
\hline \multirow{2}{*}{ Desempeño Docente } & Nunca & A veces & Siempre & Total \\
\multirow{2}{*}{ Malo } & Recuento esperado & 2.6 & 1.8 & 0.6 & 5.0 \\
& \% del total & $5.3 \%$ & $0.0 \%$ & $0.0 \%$ & $5.3 \%$ \\
\multirow{2}{*}{ Regular } & Recuento esperado & 15.6 & 10.5 & 3.8 & 30.0 \\
& \% del total & $17.0 \%$ & $12.8 \%$ & $2.1 \%$ & $31.9 \%$ \\
\multirow{2}{*}{ Bueno } & Recuento esperado & 30.8 & 20.7 & 7.5 & 59.0 \\
& \% del total & $29.8 \%$ & $22.3 \%$ & $10.6 \%$ & $62.8 \%$ \\
\multirow{2}{*}{ Total } & Recuento esperado & 49.0 & 33.0 & 12.0 & 94.0 \\
& \% del total & $52.1 \%$ & $35.1 \%$ & $12.8 \%$ & $100.0 \%$ \\
\hline
\end{tabular}

Se observa que la mayoría de los estudiantes - 29.8\% — calificó el desempeño docente como bueno, y a su vez marcaron la alternativa nunca respecto al aprendizaje basado en el enfoque superficial; mientras que ningún estudiante indicó que el desempeño docente es malo. La alternativa de nunca mostró mayor porcentaje, con 5.3\%. El aprendizaje basado en el enfoque superficial genera un conocimiento que no es duradero para el estudiante, por lo que una percepción baja de los estudiantes respecto al desempeño docente en cuanto a este indicador muestra que ellos consideran que los docentes no propician este tipo de aprendizaje, por no ser adecuado para la formación profesional.

Tabla 3

Resultados del desempeño docente y la dimensión enfoque profundo

\begin{tabular}{llllll}
\hline \multicolumn{5}{l}{ Desempeño Docente } & \multicolumn{4}{l}{ Enfoque Profundo } & \\
\multirow{2}{*}{ Malo } & Recuento esperado & 0.9 & 2.7 & 1.5 & 5.0 \\
& \% del total & $3.2 \%$ & $2.1 \%$ & $0.0 \%$ & $5.3 \%$ \\
\multirow{2}{*}{ Regular } & Recuento esperado & 5.1 & 16.0 & 8.9 & 30.0 \\
& \% del total & $8.5 \%$ & $22.3 \%$ & $1.1 \%$ & $31.9 \%$ \\
\multirow{2}{*}{ Bueno } & Recuento esperado & 10.0 & 31.4 & 17.6 & 59.0 \\
& \% del total & $5.3 \%$ & $28.7 \%$ & $28.7 \%$ & $62.8 \%$ \\
\multirow{2}{*}{ Total } & Recuento esperado & 16.0 & 50.0 & 28.0 & 94.0 \\
& \% del total & $17.0 \%$ & $53.2 \%$ & $29.8 \%$ & $100.0 \%$ \\
\hline
\end{tabular}

En la tabla 3 se muestran los resultados entre el desempeño docente y la dimensión 
enfoque profundo. La prueba de correlación de Pearson muestra como resultado $\mathrm{r}=0.731$, lo que indica que entre el desempeño docente y la dimensión enfoque profundo del aprendizaje de los estudiantes existe un grado de correlación fuerte, es decir que, a mayor desempeño docente, mayor probabilidad de que los estudiantes utilicen el enfoque profundo en su aprendizaje.

Se observa que el mismo porcentaje de estudiantes $-28.7 \%$ - marcó las opciones a veces y siempre, al considerar que el aprendizaje recibido es bueno. Así mismo, un 2.1\% consideró la opción a veces en el desempeño malo. Los estudiantes consideran que los docentes cumplen con una función formadora óptima, al fomentar el aprendizaje basado en el enfoque profundo, con el que los estudiantes pueden adquirir verdadero conocimiento significativo, que va a ser la base de su desempeño personal y profesional.

En la tabla 4 se observan los resultados del análisis del desempeño docente y la dimensión enfoque de alto rendimiento. La prueba de correlación de Pearson muestra como resultado $\mathrm{r}=0.878$, lo que indica que entre el desempeño docente y la dimensión enfoque de alto rendimiento del aprendizaje de los estudiantes existe un grado de correlación muy fuerte, es decir que, a mayor desempeño docente, mayor probabilidad de que los estudiantes utilicen el enfoque de alto rendimiento dentro de su aprendizaje.

Tabla 4

Resultados del desempeño docente y la dimensión enfoque de alto rendimiento

\begin{tabular}{llllll}
\hline & \multicolumn{4}{l}{ Enfoque de Alto rendimiento } \\
\hline Desempeño docente & & Nunca & A veces & Siempre & Total \\
\cline { 2 - 5 } Malo & Recuento esperado & 0.8 & 2.2 & 2.0 & 5.0 \\
& \% del total & $3.2 \%$ & $2.1 \%$ & $0.0 \%$ & $5.3 \%$ \\
\multirow{2}{*}{ Regular } & Recuento esperado & 4.8 & 13.1 & 12.1 & 30.0 \\
& \% del total & $5.3 \%$ & $23.4 \%$ & $3.2 \%$ & $31.9 \%$ \\
Bueno & Recuento esperado & 9.4 & 25.7 & 23.9 & 59.0 \\
\multirow{2}{*}{ Total } & \% del total & $7.4 \%$ & $18.1 \%$ & $37.2 \%$ & $62.8 \%$ \\
& Recuento esperado & 15.0 & 41.0 & 38.0 & 94.0 \\
\hline
\end{tabular}

Se observa que el $37.2 \%$ de los estudiantes evalúa de manera positiva el desempeño docente, respecto al aprendizaje adquirido, ya que lo considera como bueno. Por otro lado, la percepción sobre el desempeño malo mostró un $2.1 \%$, que consideró la opción a veces. A pesar de que en la actualidad no se fomenta de manera explícita el aprendizaje basado en un enfoque de alto rendimiento, existen aspectos culturales y académicos que implícitamente lo siguen alentando, lo cual se observa en la percepción de los estudiantes. Esto se puede deber a una tendencia de los docentes a indicarles que deben exhibir alto rendimiento en sus clases. Dicho resultado también está influenciado por los estándares que se exigen para la contratación de personal, los cuales aún siguen teniendo como premisa el alto rendimiento académico de los egresados.

\section{Discusión}

Según la prueba de correlación de Pearson, el coeficiente de correlación entre las dos 
variables es $r=0.617$, lo que indica que existe una relación fuerte entre el desempeño docente y el nivel de aprendizaje de los estudiantes de la muestra. Al ser una relación positiva, se infiere que mientras mejore el desempeño docente, mayor será el aprendizaje de los estudiantes. Este resultado es consistente con lo obtenido por Palomino (2012), quien trabajó con una muestra de 345 estudiantes de la Universidad Nacional Mayor de San Marcos - Perú-, a quienes se les aplicó un cuestionario con escala de medición tipo Likert, en el que se midieron cuatro dimensiones: estrategias didácticas, materiales didácticos, capacidades pedagógicas y responsabilidad en el desempeño de funciones. Concluyó que existe relación entre el desempeño docente y el aprendizaje de los estudiantes y que las estrategias didácticas son las que más impactan y se reflejan en las calificaciones de las clases teóricas y prácticas.

Por otro lado, los resultados contrastan con lo reportado por Acosta (2017) al estudiar la correlación entre el desempeño docente y el aprendizaje de matemáticas en estudiantes de cuarto grado de primaria. Concluyó que la relación era moderada y significativa. De igual manera, Gómez et al. (2011) investigaron la correlación entre la práctica docente y rendimiento académico de un grupo de estudiantes de medicina de la Universidad del Noreste -México- L Llegaron a la conclusión de que no existe correlación entre la práctica docente evaluada por los alumnos y su rendimiento académico. Además, identificaron que, cuando el estudiante hace la evaluación, por lo general carece de seriedad, resultado que también contrasta con el obtenido en la presente investigación.

Es claro que, de manera general, el desempeño de la labor docente influye de forma directa sobre el rendimiento de los estudiantes, ya que la base de dicho desempeño es la preparación constante y el uso de técnicas asertivas. Rahmatullah (2016) también llegó a la misma conclusión en un estudio realizado en Indonesia, donde encontró que existe una relación entre la eficacia del aprendizaje y el rendimiento de los docentes, y que además existe relación entre la competencia de los docentes y su rendimiento. Así mismo, concluye que el aumento del rendimiento de los docentes puede darse a partir de modelos que impliquen la eficacia del aprendizaje y la competencia de los docentes. En ese orden, Santos (2012), señaló:

La existencia de un marco conceptual apropiado y ampliamente aceptado acerca de qué es un buen desempeño docente, es requisito indispensable para desarrollar un sistema de evaluación que sea útil para apoyar a los profesores. Los estándares de desempeño docente que se han generado ya en otros países pueden servir de inspiración y punto de arranque para un ejercicio de reflexión colectiva respecto de qué ha de entenderse por una "buena enseñanza" y cómo ha de reconocerse. (p. 203)

Otros trabajos, como el de Martínez-Chairez et al. (2016), al analizar el desempeño docente y la calidad educativa desde la perspectiva de los profesores, concluyeron que la calidad educativa no depende únicamente del desempeño docente, sino que existen cuatro factores - escuela, contexto, docente y gobierno- que necesitan trabajar de forma colaborativa para lograr un aprendizaje verdaderamente significativo. En este mismo contexto, Gálvez y Milla (2018) concluyeron que se deben adecuar los modelos de evaluación del desempeño de los docentes, para fortalecer la profesión y, por ende, mejorar el proceso de enseñanza-aprendizaje. En relación con lo anterior, se puede mencionar una de las teorías 
que sustentan el modelo de calidad, como la creada en 1924 por Walter Andrew Shewhart (Quién es quién. Walter Andrew Shewhart., 2014), el cual creó el ciclo PHVA —planear, hacer, verificar y actuar-. El ciclo PHVA es una herramienta de mejora continua que brinda soluciones para mantener la competitividad respecto a productos y servicios. Se utiliza para la gestión de la calidad, ya que, al ser aplicada en la política y objetivos de calidad, el éxito es mayor. Además, busca crear cultura organizacional, al plantear una metodología para resolver problemas, que permite dinamizar las relaciones entre los hombres y los procesos para mejorar los estándares. Utilizado en la educación, permitirá la mejora de su calidad.

Respecto al desempeño docente y la dimensión enfoque superficial del aprendizaje, la prueba de correlación de Pearson muestra como resultado $r=-0.335$, lo que indica que entre ambas variables hay un grado de correlación moderado. Este resultado es consistente con el reportado por Díaz y Pérez (2013), quienes, al estudiar la autoeficacia y los enfoques y estrategias de aprendizaje, obtuvieron una correlación inversa entre la autoeficacia y el enfoque superficial del aprendizaje, lo cual indica que este enfoque es negativo para el proceso de aprendizaje.

En la correlación entre el desempeño docente y el enfoque profundo de aprendizaje, la prueba de Pearson muestra como resultado $\mathrm{r}=0.731$, lo que indica que entre el desempeño docente y la dimensión enfoque profundo del aprendizaje de los estudiantes existe un grado de correlación muy alta y positiva. Esto es consistente con lo obtenido por Díaz y Pérez (2013), quienes observaron correlaciones altas entre la adopción de un enfoque profundo de aprendizaje y la utilización de estrategias de disposición al aprendizaje y metacognitivas. También notaron una correlación muy alta entre dicho enfoque y la utilización de estrategias cognitivas, lo que evidentemente guarda una relación directa con lo observado en la presente investigación. El alumno que sigue este enfoque intenta relacionar los contenidos con contextos personales significativos o con conocimientos previos y encuentra el aprendizaje emocionalmente satisfactorio.

El grado de correlación entre el desempeño docente y la dimensión enfoque de alto rendimiento del aprendizaje fue de $\mathrm{r}=0.878$, según la prueba de Pearson, lo que indica una correlación muy alta y positiva, es decir que, a mayor desempeño docente, mayor probabilidad de que los estudiantes utilicen el enfoque de alto rendimiento dentro de su aprendizaje. Este resultado también fue observado por del Álamo (2015), al tomar como muestra a estudiantes de la maestría en Ciencias Militares de la Escuela Superior de Guerra del Ejército de Perú en 2014. Concluye que el desempeño docente tiene relación directa con el logro del aprendizaje, sobre todo al tratarse de la dimensión alto rendimiento. Igualmente, Monroy (2012), al estudiar el desempeño docente y el rendimiento académico en matemáticas, menciona que la dimensión alto rendimiento muestra una correlación alta con respecto al desempeño docente observado, lo que es consistente con el resultado de la presente investigación.

Lo obtenido muestra la importancia del desempeño del docente en el logro de un aprendizaje significativo, visto desde la perspectiva de los estudiantes. Esto ha sido estudiado y analizado por diferentes investigadores, que han aportado al conocimiento de este aspecto, fundamental para el desarrollo de procesos de enseñanza-aprendizaje eficientes. En tal sentido, Cortés et al. (2014), al estudiar el desempeño docente desde la perspectiva 
de estudiantes, observaron que

al estudiante principalmente le interesa lo que logra el docente con ellos, y secundariamente otros aspectos, lo que resulta novedoso, pues la mayoría de las evaluaciones, se centran en lo que hace el docente, no en sus logros con el estudiante. (p. 10)

Así mismo, Basto (2017), al realizar un estudio documental y descriptivo sobre la función docente y el rendimiento académico, destaca que las posturas metodológicas más utilizadas para medir el desempeño docente y el rendimiento de los estudiantes se centran, en su mayoría, en la postura positivista, con el empleo de complejos métodos numéricos y estadísticos. Eso deja en un segundo plano los aspectos cualitativos, que han sido poco estudiados - con excepción de algunos estudios de caso, observación y grupos focales-, lo cual deja un campo de estudio abierto.

Lo anterior también puede ser sustentado en lo obtenido por Bazar y Kraft (2017), quienes analizaron los efectos de los maestros y de la enseñanza en las actitudes y comportamientos de los estudiantes. Concluyeron que las actitudes y comportamientos de los estudiantes son predichos por las prácticas de enseñanza más cercanas a estas medidas, incluyendo el apoyo emocional y la organización del salón de clases. Sin embargo, los maestros que son efectivos en mejorar los resultados de las pruebas a menudo no son igualmente efectivos en mejorar las actitudes y conductas de los estudiantes. Los hallazgos, según los autores citados, aportan evidencia empírica a una teoría bien establecida sobre la naturaleza multidimensional de la enseñanza y la necesidad de identificar estrategias para mejorar la gama completa de las habilidades de los maestros.

Un alto nivel de desempeño docente es considerado como uno de los factores indispensables para generar estudiantes con niveles de aprendizajes esperados. Según este enfoque, para el Ministerio de Educación del Perú (2015), este desempeño se logra a través de un acuerdo técnico y social entre el Estado, los docentes y la sociedad, en torno a las competencias que se espera que dominen los docentes en las etapas de su carrera profesional, con el fin de generar un exitoso aprendizaje de todos los estudiantes. Además, se considera una herramienta estratégica bajo una política integral de desarrollo docente.

Este desempeño docente - en vez de verse como una evaluación plasmada bajo la imagen de documento técnico y social, que cada vez es más aplicado en las instituciones educativas públicas bajo las directrices evaluativas del Ministerio de Educación, con el fin de garantizar el aprendizaje de los estudiantes en todas las áreas- más bien debe verse como una oportunidad para generar estudiantes con un enfoque que mejore resultados positivos durante su proceso de aprendizaje, por lo que es necesario que el docente analice cómo desarrolla su práctica docente, qué estrategias está poniendo en práctica, qué contenidos enseña — si son de interés para el estudiante- y bajo qué enfoque está aprendiendo el estudiante, para así incentivarlo, desde una visión holística transcompleja, al uso de nuevas y mejores estrategias de aprendizaje de alto rendimiento. Es importante tener en cuenta que, para generar cambios positivos en el desempeño docente y en el aprendizaje del estudiante, es indispensable la presencia de un clima institucional armonioso entre todos los agentes educativos. 


\section{Conclusiones}

Los resultados indicaron que existe una fuerte relación entre el desempeño mostrado por los docentes de los programas de Educación Inicial y Educación Primaria y el aprendizaje de los estudiantes. Se observó que dicha relación es muy fuerte y positiva respecto al enfoque de aprendizaje profundo y de alto rendimiento, por lo que se deduce que los docentes están potenciando los enfoques orientados a la adquisición de verdadero conocimiento significativo, es decir, buscan generar en los estudiantes conocimiento duradero, efectivo y que pueda ser aplicado tanto en su vida personal como en la profesional.

Además del fortalecimiento del aprendizaje profundo, se observó que los estudiantes consideran que los docentes también influyen en la búsqueda del aprendizaje basado en enfoque de alto rendimiento. Los docentes potencian el deseo -o la necesidad- de los estudiantes de conseguir altas calificaciones, lo que también puede estar influenciado por las exigencias académicas solicitadas por los empleadores, sobre todo en estudiantes de educación inicial y de primaria, como fue el caso de estudio.

En relación con el desempeño docente en cuanto al aprendizaje basado en el enfoque superficial, se observó que la relación fue moderada y negativa. Esto indica que los estudiantes consideran que los docentes no promueven este tipo de aprendizaje y que un alto desempeño docente lleva a disminuirlo, ya que no genera verdadero conocimiento, sino que solo se centra en el aprendizaje puntual y del momento, para ser olvidado en poco tiempo, situación que lleva a que se pierda y no sea de provecho para los futuros profesionales.

Es evidente que para la universidad es importante potenciar el desempeño de sus docentes, pues, como se observó, influye de forma significativa en el nivel de aprendizaje de los estudiantes y, por ende, en su desempeño futuro como profesionales. Por eso, es fundamental expandir la investigación a otras facultades, para observar si existen diferencias en este aspecto, ya que en la revisión de trabajos previos se observaron resultados que no coinciden con los obtenidos en esta investigación.

\section{Referencias}

Acosta, S. (2017). Desempeño docente y aprendizaje de matemáticas en los estudiantes de cuarto grado (tesis de maestría), Universidad César Vallejo, Lima Perú.

Álamo del, J. (2015). Relación entre desempeño docente y nivel del logro de aprendizaje de los oficiales alumnos de la maestría en ciencias militares de la Escuela Superior de Guerra Del Ejército - 2014 (Tesis de maestría). Universidad Nacional de Educación Enrique Guzmán y Valle. Lima-Perú

Basto, R. (2017). La función docente y el rendimiento académico: una aportación al estado del conocimiento. XIV Congreso Nacional de Investigación Educativa - COMIE. San Luis de Potosí, México.

Bazar, D., \& Kraft, M. (2017). Teacher and Teaching Effects on Students' Attitudes and Behaviors. Educational Evaluation and Policy Analysis, 39(1), 146-170. https://doi. org/10.3102/0162373716670260 
Cortés, E., Campos, M., \& Moreno, M. (2014). Priorización de las dimensiones de evaluación al desempeño docente por el estudiante, en tres áreas del conocimiento. Formación Universitaria, 7(2), 3-10. https://scielo.conicyt.cl/scielo.php?script=sci_ arttext\&pid=S0718-50062014000200002

Díaz, A., \& Pérez, M. (2013). Autoeficacia, enfoque de aprendizaje profundo y estrategias de aprendizaje. International Journal of Developmental and Educational Psychology, 2(1), 341-346. https://www.redalyc.org/pdf/3498/349852173023.pdf

Duckworth, A., Quinn, P., \& Tsukayama, E. (2012). What No Child Left Behind leaves behind: The roles of IQ and self-control in predicting standardized achievement test scores and report card grades. Journal of Educational Psychology, 104(2), 439-451. https://www.ncbi.nlm.nih.gov/pmc/articles/PMC3782117/

Fasce, E. (2007). Aprendizaje profundo y superficial. Revista de Educación en Ciencias de la Salud, 4(1), 7-8. http://www.udec.cl/ofem/recs/anteriores/vol412007/esq41.pdf

Freire, P., \& Faundez, A. (2013). Por una pedagogía de la pregunta. Crítica a una educación basada en respuestas a preguntas inexistentes. Siglo XXI.

Gálvez, E., \& Milla, R. (2018). Evaluación del desempeño docente: Preparación para el aprendizaje de los estudiantes en el Marco de Buen Desempeño Docente. Propósitos y Representaciones, 6(2), 407-452. https://doi.org/10.20511/pyr2018.v6n2.236

Gómez, V., Rosales, S., García, J., \& Berrones, K. (2011). Correlación entre la práctica docente y rendimiento académico en un grupo de estudiantes de medicina. Archivos en Medicina Familiar, 13(3), 117-121.

Hopkins, W. (2014). A New View of Statistics. https://complementarytraining.net/wpcontent/uploads/2013/10/Will-Hopkins-A-New-View-of-Statistics.pdf

Instituto Nacional de Estadística e Informática. (2014). Encuesta nacional a egresados universitarios y universidades. https:/www.inei.gob.pe/media/MenuRecursivo/ publicaciones_digitales/Est/Lib1298/Libro.pdf

Martínez-Chairez, G., Guevara-Araiza, A., \& Valles-Ornelas, M. (2016). El desempeño docente y la calidad educativa. Ra Ximhai, 12(6), 123-134.

Ministerio de Educación del Perú. (2015). Rutas de Aprendizaje Perú. http://www.minedu. gob.pe/rutas-delaprendizaje/documentos/Primaria/Matematica-III.pdf

Monroy, M. (2012). Desempeño docente y rendimiento académico en matemática de los alumnos de una institución educativa de Ventanilla - Callao (Tesis de maestría). Universidad San Ignacio de Loyola. Lima, Perú.

Muñoz, E., \& Gómez, J. (2005). Enfoques de aprendizaje y rendimiento académico de los estudiantes universitarios. Revista de Investigación Educativa, 23(2), 417-432.

Palomino, F. (2012). El desempeño docente y el aprendizaje de los estudiantes de la Unidad Académica de Estudios Generales de la Universidad de San Martín de Porres (Tesis de maestría). Universidad Nacional Mayor de San Marcos. Lima, Perú.

Quién es quién. Walter Andrew Shewhart (1891 Illinois, EEUU - 1967 Nueva Jersey, EEUU). 
(2014). Revista Índice. http://www.revistaindice.com/numero58/p2.pdf

Rahmatullah, M. (2016). The Relationship between Learning Effectiveness, Teacher Competence and Teachers Performance Madrasah Tsanawiyah at Serang, Banten, Indonesia. Higher Education Studies, 6(1), 169-181. https://doi.org/10.5539/hes. v6n1p169

Rueda, M. (2009). La evaluación del desempeño docente: consideraciones desde el enfoque por competencias. Revista Electrónica de Investigación Educativa, 11(22). http://redie. uabc.mx/vol11 no2/contenido-rueda3.html

Santos del Real, A. (2012). Evaluación Docente. Educación Química, 23 (2), 200-204. http:// dx.doi.org/10.1016/S0187-893X(17)30109-X

Soler, M., Cárdenas, F., \& Hernández-Piña, F. (2018). Enfoques de enseñanza y enfoques de aprendizaje: perspectivas teóricas promisorias para el desarrollo de investigaciones en educación en ciencias. Ciência \& Educação (Bauru), 24(4), 993-1012. https://doi. org/10.1590/1516-731320180040012

Soler-Contreras, M., Cárdenas-Salgado, F., Hernández-Pina, F., \& Monroy-Hernández, F. (2017). Enfoques de aprendizaje y enfoques de enseñanza: origen y evolución. Educación y Educadores, 20(1), 65-88. https://doi.org/10.5294/edu.2017.20.1.4

Usun, S. (2004). Important learning dimensions influencing undergraduate students learning and academic achievement in higher education. The Turkish Online Journal of Educational Technology - TOJET, 3(4), 15-27. https://files.eric.ed.gov/fulltext/ EJ1101914.pdf 